\title{
Components of Metabolic Syndrome in Korean Adults: A Hospital-Based Cohort at Seoul National University Bundang Hospital
}

\author{
Soo Lim ${ }^{1, *}$, Se Hee Min², Ji Hyun Lee ${ }^{3}$, Lee Kyung Kim4, Dong-Hwa Lee ${ }^{5}$, Jie-Eun Lee' ${ }^{1}$ Kyoung Min Kim', Sunmi Lee ${ }^{6}$, \\ Kyoung-Chan Park ${ }^{6,7}$, Yun Jong Lee ${ }^{1,6}$ \\ ${ }^{1}$ Department of Internal Medicine, Seoul National University Bundang Hospital, Seoul National University College of Medicine, Seongnam; ${ }^{2}$ Department of Internal \\ Medicine, Asan Medical Center, University of Ulsan College of Medicine, Seoul; ${ }^{3}$ Department of Internal Medicine, Veterans Health Service Medical Center, Seoul; \\ ${ }^{4}$ Department of Internal Medicine, Cheju Halla General Hospital, Jeju; ${ }^{5}$ Department of Internal Medicine, Chungbuk National University Hospital, Cheongju; \\ ${ }^{6}$ Human Biobank, Seoul National University Bundang Hospital, Seongnam; ${ }^{7}$ Department of Dermatology, Seoul National University Bundang Hospital, Seoul \\ National University College of Medicine, Seongnam, Korea
}

Background: The prevalence of metabolic syndrome is increasing in Korea, particularly among young adults. This trend will increase the incidence of cardiovascular and metabolic diseases in the future. Therefore, it is imperative to detect and prevent metabolic abnormalities early in life. Here, we established a hospital-based biobank cohort to identify the most prevalent dysmetabolic phenotype. The aim of this report was to inform other researchers of our protocol and to share our data for future collaboration.

Methods: The baseline examination comprised health-related questionnaires, anthropometric and handgrip strength measurements, bioelectrical impedance analysis of body composition, and nutritional assessment. Relevant biochemical parameters were measured, and oral glucose tolerance tests were performed. Metabolic syndrome was defined according to the National Cholesterol Education Program Adult Treatment Panel III criteria with Asian waist circumference criteria.

Results: From a total of about 3,000 employees aged 20 to 59 years working at Seoul National University Bundang Hospital, 1,017 were enrolled from 2015 to 2016. The mean age was $34.5 \pm 8.4$ years for men $(n=311$, $30.6 \%$ ) and $30.9 \pm 8.5$ years for women $(n=706,69.4 \%)$. The overall prevalence of metabolic syndrome was $7.6 \%$ (17.7\% in men and $3.1 \%$ in women). Among the five components of metabolic syndrome, high blood pressure was the most prevalent in both men (51.4\%) and women (13.8\%).

Conclusion: Although further follow-up data are needed, we expect that more adverse cardiovascular events may occur in men than in women. This hospital-based cohort will serve as the foundation for a comprehensive evaluation of metabolic syndrome and future cardiometabolic disease risk in middle-aged Koreans.

Key words: Metabolic syndrome, Cardiovascular disease, Risk factor, Biobank

\author{
Received February 26, 2019 \\ Reviewed March 20, 2019 \\ Accepted May 3, 2019 \\ *Corresponding author \\ Soo Lim \\ https://orcid.org/0000-0002-4137-1671 \\ Department of Internal Medicine, Seoul \\ National University Bundang Hospital, \\ Seoul National University College of \\ Medicine, 82 Gumi-ro 173beon-gil, \\ Bundang-gu, Seongnam 13620, Korea \\ Tel: +82-31-787-7035 \\ Fax: +82-31-787-4051 \\ E-mail:limsoo@snu.ac.kr
}

\section{INTRODUCTION}

Metabolic syndrome is a cluster of cardiometabolic risk factors comprising central obesity, dyslipidemia, hypertension, and hyperglycemia. ${ }^{1}$ Hence, cardiovascular events and mortality are consistently more prevalent in subjects with metabolic syndrome. ${ }^{2}$ The main pathophysiology of metabolic syndrome is insulin resistance and central obesity, although the International Diabetes Federation (IDF) diagnostic criteria emphasize central obesity ${ }^{3}$, while the World Health Organization emphasizes impaired glucose regulation and/ or insulin resistance. ${ }^{4}$ The diagnostic criteria of metabolic syndrome established by the National Cholesterol Education Program Adult

Copyright (C) 2019 Korean Society for the Study of Obesity

() This is an Open Access article distributed under the terms of the Creative Commons Attribution Non-Commercial License (http://creativecommons.org/licenses/by-nc/4.0/) which permits unrestricted non-commercial use, distribution, and reproduction in any medium, provided the original work is properly cited. 
Treatment Panel III (NCEP-ATP III) have merged these concepts ${ }^{5}$, and the IDF has published an updated set of criteria. ${ }^{6}$

The reported prevalence of metabolic syndrome varies widely according to age group, sex, ethnicity, and diagnostic criteria. ${ }^{7}$ In the Korea National Health and Nutrition Examination Survey (KNHANES), the prevalence of metabolic syndrome assessed by modified NCEP-ATP III guidelines increased from 24.9\% in 1998 to $31.3 \%$ in $2007 .{ }^{8}$ More recent KNHANES data from 2007 to 2010 revealed that the prevalence of metabolic syndrome was $31.9 \%$ in men and $29.0 \%$ in women aged 30 years and above. ${ }^{9}$ In the present report, metabolic syndrome was defined by modified NCEP-ATP III guidelines, with the definition of abdominal obesity established by the Korean Society for the Study of Obesity (waist circumference $\geq 90 \mathrm{~cm}$ in men and $\geq 85 \mathrm{~cm}$ in women). ${ }^{10}$ More strikingly, the KNHANES indicated that the prevalence of metabolic syndrome in young Koreans increased to $7.8 \%$ in 2007, whereas data from the United States revealed that the prevalence of metabolic syndrome in young people decreased to $6.5 \%$ in $2007 .{ }^{11}$ In addition, among subjects aged 20 years and above, the prevalence of metabolic syndrome in the United States was stable at $36.1 \%$ from 2007 to 2008 and $34.7 \%$ from 2011 to $2012 .{ }^{12}$ In brief, metabolic syndrome has shown an increasing trend in Koreans, in contrast to the U.S. population.

A previous survey demonstrated that Western dietary pattern and sedentary behavior were associated with a high prevalence of metabolic syndrome in the United States. ${ }^{13,14}$ However, it has been reported that Korean women do not follow typical Western dietary patterns and thus have a relatively low prevalence of metabolic syndrome. ${ }^{15}$ In terms of physical activity, excessive sitting time and low physical activity level were associated with a high risk of metabolic syndrome in Koreans. ${ }^{16,17}$ Interestingly, muscle strength was significantly lower in elderly male subjects with metabolic syndrome than in their normal counterparts. ${ }^{18}$ In addition, central obesity accompanied by sarcopenia was closely associated with metabolic syndrome in elderly Koreans. ${ }^{19}$ Therefore, a detailed assessment of nutrition, physical activity, muscle strength, and body composition is needed to provide a comprehensive understanding of metabolic syndrome in the Korean population. Furthermore, in the era of precision medicine, appropriate sample collection is necessary to confirm the many potential biomarkers that predict metabolic syn- drome and cardiovascular disease.

Building a cohort with employees in an institution or company is simple and effective in terms of recruitment and regular followup. ${ }^{20}$ Hospital-based cohorts have several advantages, including easy access to healthcare facilities and high participation and follow-up rates. ${ }^{21}$ Therefore, we established a hospital-based biobank cohort to determine the most prevalent metabolic abnormalities among metabolic syndrome components and to identify predictive biomarkers for risk of future diabetes mellitus and cardiovascular events such as nonfatal myocardial infarction, nonfatal stroke, and cardiovascular death. The primary purpose of this cohort study was to investigate the prevalence of the established risk factors for metabolic syndrome in Korean adults. The secondary purposes were to discover any new risk factors or predictors of metabolic syndrome and to uncover their possible mechanisms. We plan to follow up with this cohort every three years for up to 12 years for this purpose. Lastly, we will share our protocol in detail to merge our results with those of other hospital-based cohort studies and will release our dataset for future collaboration.

\section{METHODS}

\section{Study design}

We designed a hospital-based prospective cohort study. Subjects were recruited at Seoul National University Bundang Hospital (SNUBH), Seongnam, Korea. The subjects were hospital workers in various occupational categories: physicians, nurses, pharmacists, technicians, and other hospital personnel. Under the national health and safety laws in Korea, an annual general health examination is mandatory for all hospital employees. The components of the mandatory health examination depend on the occupational category. We enrolled subjects who underwent medical checkups for this purpose. The study protocol was approved by the Institutional Review Board of SNUBH (IRB No. B-1505/298-005). All subjects gave written informed consent to participate in our study and/or be included in the SNUBH-biobank. This study was registered at https://clinicaltrials.gov (NCT02640859).

\section{Study population and patient enrollment}

Male and female employees of SNUBH who were 20 to 59 years 
old were recruited through advertisements and enrolled voluntarily. Subjects who had any type of malignancy, previous cardiovascular events, or known end-stage renal disease requiring dialysis were excluded. In addition, subjects who were expected to change jobs within the next 6 months were excluded. We aimed to recruit 500 subjects per year for 2 years (2015-2016) to reach a target of 1,000 subjects. The baseline study was performed from 2015 to 2016 .

\section{Demographics and anthropometric measurements}

At the baseline examination, self-completed questionnaires were used to assess the subjects' demographics, general health status, and social history (alcohol consumption, smoking status, physical activity, and family history of chronic diseases). We defined ex-smokers as those who had smoked more than 100 cigarettes in their lifetime but were not smoking at present. Physical activity was divided into four categories: (1) none; (2) regular walking, which was defined as $\geq 30$ minutes of walking per day at least 3 days per week; (3) regular exercise, which included $\geq 30$ minutes of activities such as fast walking, tennis, regular cycling, and moderate-intensity daily activities such as mopping at least 3 days per week; and (4) vigorous exercise, which included running, fast cycling, and climbing for $>20$ minutes at least 3 days per week. The parity of women was assessed with a standard questionnaire, which included the number of live births, year of delivery, and method of delivery.

Height and body weight were measured on an electronic scale with the subjects wearing light indoor clothing. Body mass index (BMI) was calculated as weight $(\mathrm{kg})$ divided by height squared $\left(\mathrm{m}^{2}\right)$. Waist circumference was measured with a measuring tape at the midline between the lowest rib margin and the iliac crest. Systolic and diastolic blood pressures were measured with an electronic blood pressure meter (UA-1020 device; A\&D, Tokyo, Japan) while the subjects were seated. Blood pressure was measured twice at a 5-minute interval, and the mean value was used in the analysis.

\section{Nutritional analysis}

We performed a nutritional assessment using a web-based, computer-assisted personal interview system, which has been confirmed to reflect individual usual nutrient intake. ${ }^{22}$ We provided information on how to access the website and instructed participants to enter their nutrient intakes during 2 weekdays and 1 weekend day. After the survey, we informed the participants of their analyzed results and provided dietary recommendations based on their nutritional status.

\section{Bioelectrical impedance analysis}

After subjects had fasted overnight and urinated, a trained nurse measured their body composition (fat and muscle mass) with a bioelectrical impedance analysis (BIA) machine (InBody 720 or InBody770; InBody, Seoul, Korea) while the subjects wore light clothing, on the same day as blood sampling. The InBody720 and InBody770 use the same hardware for production of impedance. Water intake was restricted before each BIA measurement. Participants were placed in an upright position with bare feet, and an instructor entered their identification number, age, sex, and height into the machine. The participants were then instructed to grasp the electrode bars with both hands and to separate their arms from their trunk. In this manner, the subjects' feet and hands made contact with eight electrodes. After the machine was started by the instructor, six different frequencies $(1,5,50,250,500$, and 1,000 $\mathrm{kHz}$ ) were used on five segments of the body: both arms and legs and the trunk. Abdominal visceral fat area was estimated through the manufacturer's calculation method. ${ }^{23}$

\section{Handgrip strength}

Handgrip strength was measured with an electronic grip strength dynamometer (TKK 5401, GripD; Takei Scientific Instruments, Tokyo, Japan) while the subjects were seated. Subjects were asked to perform maximum force trials twice with each hand in a rightthen-left sequence. The mean values of repeated measures were recorded.

\section{Biochemical data}

We instructed the participants not to eat food after midnight. Baseline blood samples were drawn after at least 10 hours of fasting, and the collected samples were centrifuged at 3,000 rpm for 10 minutes at $4^{\circ} \mathrm{C}$. Plasma glucose level was analyzed by the hexokinase method, while high-density lipoprotein (HDL) cholesterol and low-density lipoprotein cholesterol levels were measured by homogeneous enzymatic assays, and triglyceride level was measured by a glycerol-3-phosphate oxidase peroxide method. An XE- 
2100 Hematology Analyzer (Sysmex Corp., Kobe, Japan) was used to measure white blood cell count, hemoglobin level, hematocrit level, and platelet count. Aspartate and alanine aminotransferase (NADH-UV method), total bilirubin (bilirubin oxidase method), thyroid function (electrochemiluminescence immunoassay), blood urea nitrogen (urease/glutamate dehydrogenase method), creatinine (Jaffe's kinetic method), calcium (o-cresolphtalein chromogenic method), and phosphorus (molybdate reduction method) levels were measured at the central laboratory of SNUBH. The biochemical tests were performed immediately after sample collection. Aliquots of serum, plasma, whole blood, DNA, and RNA (PAXgene Blood RNA System, Qiagen, Hilden, Germany) were kept in deep freezers until tested $\left(-80^{\circ} \mathrm{C}\right)$.

\section{Oral glucose tolerance test}

We performed a standard 75-g 2-hour oral glucose tolerance test (OGTT) on selected subjects who agreed to participate. All participants were advised to continue their regular diet and to avoid excessive alcohol consumption and vigorous exercise for at least 3 days before the test. They were asked to ingest $75 \mathrm{~g}$ of glucose in a 225-mL volume (Glu Orange; McNulty Pharmaceutical, Seoul, Korea) over 5 minutes after a baseline blood sample was drawn. ${ }^{24}$ Aliquots of $4 \mathrm{~mL}$ of blood were collected 30 and 120 minutes after glucose ingestion for measurements of postload glucose concentration (Postload30 and Postload120, respectively). The blood samples were kept on ice during the tests, and the centrifugation procedure was the same as above.

\section{Definition of metabolic syndrome}

Metabolic syndrome was defined according to the IDF metabolic syndrome criteria ${ }^{6}$, and waist circumference data were evaluated based on Asia-Pacific abdominal obesity criteria. ${ }^{10}$ Metabolic syndrome was diagnosed by the presence of abdominal obesity (waist circumference $\geq 90 \mathrm{~cm}$ in men and $\geq 85 \mathrm{~cm}$ in women), plus two or more of the following factors: (1) blood triglyceride level $\geq 150 \mathrm{mg} / \mathrm{dL}$ or specific treatment for this lipid abnormality; (2) HDL-cholesterol level $<40 \mathrm{mg} / \mathrm{dL}$ in men and $<50 \mathrm{mg} / \mathrm{dL}$ in women or specific treatment for this lipid abnormality; (3) blood pressure $\geq 130 / 85 \mathrm{mmHg}$ or use of antihypertensive medications; and (4) fasting blood glucose $\geq 100 \mathrm{mg} / \mathrm{dL}$ or use of antidiabetic medications.

\section{Statistical analysis}

The prevalence of metabolic syndrome in Korea is estimated to be $5.2 \%-31.7 \%$ among men and $9.0 \%-40.1 \%$ among women. The applied criteria for metabolic syndrome and the age range of the participants are important factors affecting the incidence of cardiometabolic diseases. In our cohort, we planned to recruit subjects aged 20-59 years, so the expected prevalence of metabolic syndrome was $10 \%-20 \%$ in both sexes, with a higher prevalence in men than in women. On the assumption of a cardiometabolic event rate of $10 \%$ in women and $20 \%$ in men over 3 years, we determined that a total of 1,000 participants ( 700 women and $300 \mathrm{men}$ ) would be required for statistical analysis and sampling convenience. We planned to recruit around 1,000 subjects with a two to one ratio of women to men from 2015 to 2016.

All data are expressed as the mean and standard deviation (SD) or as number and percentage. The Kolmogorov-Smirnov test was applied to evaluate the normality of variable distributions. In the case of continuous variables that were not normally distributed, we performed nonparametric analyses. Before variables were compared between the two sexes, differences in variance were subjected to Levene's test. Correlations between variables were analyzed by Pearson's test. Categorical variables were compared by chi-square tests. For continuous variables, means were compared by Student $\mathrm{t}$-test or nonparametric t-test. The analyses were performed with IBM SPSS version 20.0 (IBM Corp., Armonk, NY, USA). For all tests, $P<0.05$ was considered to be statistically significant.

\section{RESULTS}

\section{Baseline characteristics}

Supplementary Table 1 displays the items measured in the baseline examination. Table 1 lists the clinical and biochemical characteristics of the subjects. At baseline, 1,017 subjects were enrolled. The mean age of the subjects $( \pm S D)$ was $32.0 \pm 8.6$ years, and the men were older than the women ( $34.5 \pm 8.4$ years vs. $30.9 \pm 8.5$ years, $P<0.001)$. In this cohort, $69.4 \%$ were women. The mean waist circumference was $85.0 \pm 8.9 \mathrm{~cm}$ in men and $70.4 \pm 7.9 \mathrm{~cm}$ in women. Systolic blood pressure was higher in men than in women: $127.1 \pm$ $11.8 \mathrm{mmHg}$ in men vs. $112.9 \pm 10.9 \mathrm{mmHg}$ in women $(P<0.001)$. Plasma triglyceride level was also significantly different between 
Table 1. Clinical and biochemical characteristics of the enrolled subjects

\begin{tabular}{|c|c|c|c|c|}
\hline Variable & Total $(n=1,017)$ & $\operatorname{Men}(n=311)$ & Women $(n=706)$ & $P$ \\
\hline Age (yr) & $32.0 \pm 8.6$ & $34.5 \pm 8.4$ & $30.9 \pm 8.5$ & $<0.001$ \\
\hline Height (cm) & $165.2 \pm 7.9$ & $173.7 \pm 6.0$ & $161.5 \pm 5.3$ & $<0.001$ \\
\hline Body weight (kg) & $61.1 \pm 12.4$ & $74.2 \pm 10.9$ & $55.4 \pm 7.8$ & $<0.001$ \\
\hline $\mathrm{BMI}\left(\mathrm{kg} / \mathrm{m}^{2}\right)$ & $22.2 \pm 3.3$ & $24.6 \pm 3.1$ & $21.2 \pm 2.8$ & $<0.001$ \\
\hline Waist circumference $(\mathrm{cm})$ & $74.8 \pm 10.6$ & $85.0 \pm 8.9$ & $70.4 \pm 7.9$ & $<0.001$ \\
\hline Systolic blood pressure (mmHg) & $117.3 \pm 13.0$ & $127.1 \pm 11.8$ & $112.9 \pm 10.9$ & $<0.001$ \\
\hline Diastolic blood pressure (mmHg) & $73.4 \pm 11.7$ & $80.4 \pm 9.8$ & $70.3 \pm 11.1$ & $<0.001$ \\
\hline WBC $\left(10^{3} / \mu L\right)$ & $6.2 \pm 2.4$ & $6.1 \pm 1.5$ & $6.2 \pm 2.7$ & 0.653 \\
\hline Hemoglobin (g/dL) & $13.9 \pm 1.5$ & $15.5 \pm 1.0$ & $13.2 \pm 1.0$ & $<0.001$ \\
\hline Hematocrit (\%) & $42.0 \pm 4.0$ & $46.0 \pm 2.8$ & $40.1 \pm 3.0$ & $<0.001$ \\
\hline Platelets $\left(10^{3} / \mu \mathrm{L}\right)$ & $265.0 \pm 55.5$ & $257.4 \pm 50.3$ & $268.6 \pm 57.6$ & 0.006 \\
\hline Total cholesterol (mg/dL) & $186.5 \pm 31.2$ & $193.0 \pm 35.5$ & $183.7 \pm 28.8$ & $<0.001$ \\
\hline Triglycerides (mg/dL) & $93.1 \pm 71.3$ & $131.3 \pm 107.3$ & $76.7 \pm 37.8$ & $<0.001$ \\
\hline HDL cholesterol (mg/dL) & $64.0 \pm 15.4$ & $54.9 \pm 12.9$ & $67.9 \pm 14.7$ & $<0.001$ \\
\hline LDL cholesterol (mg/dL) & $102.8 \pm 28.7$ & $112.5 \pm 34.2$ & $99.3 \pm 25.5$ & $<0.001$ \\
\hline Fasting plasma glucose (mg/dL) & $90.6 \pm 13.8$ & $95.5 \pm 18.9$ & $88.5 \pm 10.3$ & $<0.001$ \\
\hline Postload30 (mg/dL, n=222) & $144.3 \pm 36.3$ & $164.2 \pm 54.1$ & $138.2 \pm 26.1$ & $<0.001$ \\
\hline Postload120 (mg/dL, $\mathrm{n}=218$ ) & $107.4 \pm 36.1$ & $119.0 \pm 61.7$ & $103.7 \pm 21.5$ & 0.007 \\
\hline AST (IU/L) & $20.4 \pm 7.3$ & $24.8 \pm 8.8$ & $18.7 \pm 5.9$ & $<0.001$ \\
\hline ALT (IU/L) & $17.6 \pm 12.5$ & $28.1 \pm 17.5$ & $13.5 \pm 6.1$ & $<0.001$ \\
\hline$\gamma \mathrm{GT}(\mathrm{IU} / \mathrm{L})$ & $21.1 \pm 28.4$ & $35.9 \pm 47.0$ & $14.8 \pm 8.6$ & $<0.001$ \\
\hline Total bilirubin (mg/dL) & $0.8 \pm 0.3$ & $0.9 \pm 0.3$ & $0.7 \pm 0.3$ & $<0.001$ \\
\hline Total T3 (ng/mL) & $1.12 \pm 0.19$ & $1.14 \pm 0.20$ & $1.11 \pm 0.19$ & 0.072 \\
\hline Free T4 (ng/dL) & $1.26 \pm 0.18$ & $1.32 \pm 0.21$ & $1.23 \pm 0.17$ & $<0.001$ \\
\hline $\mathrm{TSH}(\mathrm{IU} / \mathrm{mL})$ & $2.2 \pm 1.7$ & $2.1 \pm 1.4$ & $2.2 \pm 1.8$ & 0.159 \\
\hline BUN (mg/dL) & $11.9 \pm 3.1$ & $13.5 \pm 3.0$ & $11.2 \pm 2.9$ & $<0.001$ \\
\hline Creatinine (mg/dL) & $0.72 \pm 0.16$ & $0.89 \pm 0.12$ & $0.64 \pm 0.12$ & $<0.001$ \\
\hline $\mathrm{eGFR}\left(\mathrm{mL} / \mathrm{min} / 1.73 \mathrm{~m}^{2}\right)$ & $108.4 \pm 20.8$ & $99.3 \pm 16.2$ & $112.6 \pm 21.4$ & $<0.001$ \\
\hline Calcium (mg/dL) & $9.1 \pm 0.3$ & $9.2 \pm 0.3$ & $9.1 \pm 0.3$ & $<0.001$ \\
\hline Phosphorous (mg/dL) & $3.7 \pm 0.5$ & $3.5 \pm 0.5$ & $3.7 \pm 0.5$ & $<0.001$ \\
\hline
\end{tabular}

Values are presented as mean \pm standard deviation.

BMI, body mass index; WBC, white blood cell; HDL, high-density lipoprotein; LDL, low-density lipoprotein; Postload30 and Postload120, postload glucose levels 30 and 120 minutes after the oral glucose tolerance test; AST, aspartate aminotransferase; ALT, alanine aminotransferase; $\gamma \mathrm{GT}$, $\gamma$-glutamyl transferase; T3, triiodothyronine; T4, thyroxin; TSH, thyroid stimulating hormone; BUN, blood urea nitrogen; eGFR, estimated glomerular filtration rate.

men and women: $131.3 \pm 107.3 \mathrm{mg} / \mathrm{dL}$ in men versus $76.7 \pm 37.8$ $\mathrm{mg} / \mathrm{dL}$ in women $(P<0.001)$. The mean fasting plasma glucose level was $95.5 \pm 18.9 \mathrm{mg} / \mathrm{dL}$ in men and $88.5 \pm 10.3 \mathrm{mg} / \mathrm{dL}$ in women $(P<0.001)$. During the OGTT, the Postload30 level was highest $(144.3 \pm 36.3 \mathrm{mg} / \mathrm{dL})$, followed by the Postload120 level $(107.4 \pm 36.1 \mathrm{mg} / \mathrm{dL})$ and the fasting level. The Postload30 and Postload120 levels were higher in men than in women $(P<0.01)$.

Table 2 displays the medical and family histories of the subjects. The proportion of current smokers was $9.3 \%$ in men and $0.1 \%$ in women $(P<0.001)$. Substantial alcohol consumption $(\geq 30 \mathrm{~g} /$ day for men or $\geq 20 \mathrm{~g} /$ day for women) was observed in $14.5 \%$ of men and 5.4\% of women. In the physical activity assessment, $16.1 \%$ of men and $21.7 \%$ of women exercised regularly. Among all participants, $16.6 \%$ and $10.4 \%$ of the subjects had family histories of hypertension and diabetes mellitus, respectively.

\section{Metabolic syndrome components}

In total, 77 subjects (7.6\%) met the criteria for metabolic syndrome. There was a significant difference in prevalence of metabolic syndrome between the sexes: $17.7 \%$ in men and $3.1 \%$ in women. All individual components of metabolic syndrome were also more prevalent in men than in women (Table 3). When we compared 
Table 2. Past and current medical conditions, social factors, and family histories

\begin{tabular}{|c|c|c|c|c|}
\hline Variable & Total (\%) & Men $(\%)$ & Women (\%) & $P^{*}$ \\
\hline Antihypertensive medication (yr) & 3.2 & 6.8 & 1.4 & $<0.001$ \\
\hline $20-29$ & 0.2 & 1.0 & 0.3 & \\
\hline $30-39$ & 2.4 & 4.5 & 1.0 & \\
\hline $40-49$ & 5.4 & 10.9 & 1.4 & \\
\hline $50-59$ & 24.1 & 38.1 & 16.2 & \\
\hline Antidiabetic medication (yr) & 0.8 & 2.3 & 0.1 & 0.001 \\
\hline $20-29$ & 0.0 & 0.0 & 0.0 & \\
\hline 30-39 & 0.3 & 0.8 & 0.0 & \\
\hline $40-49$ & 0.8 & 1.8 & 0.0 & \\
\hline $50-59$ & 10.3 & 23.8 & 2.7 & \\
\hline Lipid-lowering medication (yr) & 3.1 & 6.1 & 1.8 & 0.001 \\
\hline $20-29$ & 0.8 & 1.0 & 0.8 & \\
\hline $30-39$ & 2.4 & 4.5 & 1.0 & \\
\hline $40-49$ & 7.0 & 10.9 & 4.1 & \\
\hline $50-59$ & 19.0 & 28.6 & 13.5 & \\
\hline Alcohol consumption & & & & $<0.001$ \\
\hline Non-drinker & 30.4 & 18.0 & 35.8 & \\
\hline Current drinker & 69.6 & 82.0 & 64.2 & \\
\hline $\begin{array}{l}\text { Substantial alcohol consumption } \\
\qquad(\geq 30 \mathrm{~g} / \text { day in men, } \geq 20 \mathrm{~g} / \text { day in women) }\end{array}$ & 8.2 & 14.5 & 5.4 & $<0.001$ \\
\hline Smoking status & & & & $<0.001$ \\
\hline Non-smoker & 93.6 & 81.4 & 99.0 & \\
\hline Ex-smoker & 3.4 & 9.3 & 0.8 & \\
\hline Current smoker & 2.9 & 9.3 & 0.1 & \\
\hline Regular exercise, yes & 20.0 & 16.1 & 21.7 & 0.041 \\
\hline \multicolumn{5}{|l|}{ Family history } \\
\hline Hypertension & 16.6 & 12.5 & 18.4 & 0.022 \\
\hline Diabetes mellitus & 10.4 & 8.0 & 11.5 & 0.119 \\
\hline Dyslipidemia & 7.6 & 5.5 & 8.5 & 0.096 \\
\hline Cardiovascular disease & 11.4 & 2.6 & 4.8 & 0.123 \\
\hline Cerebrovascular disease & 5.3 & 4.2 & 5.8 & 0.362 \\
\hline
\end{tabular}

${ }^{*} P$-values are shown for comparison of variables between men and women.

the components of metabolic syndrome in women according to parity, all values were higher in women who had been pregnant than in those who had not (Supplementary Table 2). After the evaluation, we recommended lifestyle improvements to participants who had symptoms of metabolic syndrome. In men, high blood pressure was the most prevalent factor (51.4\%), followed by abdominal obesity (28.3\%) and high triglyceride level (26.9\%). The prevalences of high blood glucose and low HDL cholesterol were $23.7 \%$ and $8.6 \%$, respectively.

In women, all individual components of metabolic syndrome were uncommon. High blood pressure was the most prevalent abnormality (13.8\%), followed by hyperglycemia (12.3\%) and low
HDL cholesterol levels (7.6\%). The prevalences of abdominal obesity (defined by a waist circumference $\geq 85 \mathrm{~cm}$ ) and hypertriglyceridemia were $6.5 \%$ and $4.6 \%$, respectively (Fig. 1). The rates of abnormal glucose and blood pressure measurements increased with age in both sexes.

\section{Body composition data}

Body composition data (as assessed by BIA) are shown in Table 4 . Lean body mass was higher in men than in women $(53.5 \pm 6.8 \mathrm{~kg}$ vs. $36.5 \pm 3.9 \mathrm{~kg}, P<0.001)$. Whole body fat percentage was significantly lower in men than in women $(23.2 \% \pm 5.8 \%$ vs. $29.5 \% \pm$ $5.5 \%, P<0.001)$. High body fatness, defined as a whole-body fat percentage $\geq 25 \%$ in men and $\geq 30 \%$ in women, was found in $35.9 \%$ of men and $45.8 \%$ of women. Abdominal visceral fat area estimated by BIA was larger in men than in women $\left(99.9 \pm 42.0 \mathrm{~cm}^{2}\right.$ vs. $\left.58.9 \pm 40.2 \mathrm{~cm}^{2}, P<0.001\right)$. Abdominal visceral obesity, defined as abdominal visceral fat area $\geq 100 \mathrm{~cm}^{2}$, was three times more prevalent in men than in women $(P<0.001)$.

\section{Handgrip strength}

Handgrip strength of the dominant hand differed significantly between the sexes $(P<0.001): 41.2 \pm 7.9 \mathrm{~kg}$ in men and $24.1 \pm 4.9 \mathrm{~kg}$ in women (Table 4). Weak handgrip strength, defined as lower than the 30th percentile of age- and sex-specific reference data $(<40 \mathrm{~kg}$ in men and $<25 \mathrm{~kg}$ in women $)^{25}$, was found in $42.1 \%$ of men and $56.9 \%$ of women.

In the correlation analysis, in both men and women, handgrip strength correlated positively with muscle mass $(r=0.589$ and $r=$ 0.397 , respectively, both $P<0.001)$ and negatively with body fat percentage $(\mathrm{r}=-0.220, P<0.001$ and $\mathrm{r}=-0.096, P=0.017$, respectively).

In simple correlation analysis, there was no significant correlation between handgrip strength and number of metabolic syndrome components. However, in partial correlation analysis adjusted for BMI, there was a significant negative correlation between handgrip strength and number of metabolic syndrome components in both men and women $(\mathrm{r}=-0.139, P=0.025$ and $\mathrm{r}=-0.127, P=0.002$, respectively). 
Table 3. Mean value of each component of metabolic syndrome and prevalence of metabolic syndrome according to sex and age group

\begin{tabular}{|c|c|c|c|c|c|c|c|}
\hline Variable & $W C(\mathrm{~cm})$ & $\mathrm{SBP}(\mathrm{mmHg})$ & $\mathrm{DBP}(\mathrm{mmHg})$ & $\mathrm{TG}(\mathrm{mg} / \mathrm{dL})$ & HDL cholesterol (mg/dL) & $\mathrm{FPG}(\mathrm{mg} / \mathrm{dL})$ & MetS (\%) \\
\hline \multicolumn{8}{|l|}{ Men (yr) } \\
\hline $20-29(n=103)$ & 83.1 & 125.9 & 77.7 & 114.5 & 56.8 & 91.9 & 7.8 \\
\hline $30-39(n=132)$ & 85.5 & 126.6 & 80.7 & 119.2 & 54.9 & 93.3 & 15.2 \\
\hline $40-49(n=55)$ & 86.8 & 129.3 & 84.7 & 191.1 & 50.1 & 102.1 & 36.4 \\
\hline $50-59(n=21)$ & 86.2 & 131.0 & 81.2 & 133.1 & 58.4 & 110.0 & 33.3 \\
\hline Total $(n=311)$ & 85.0 & 127.1 & 80.4 & 131.3 & 54.9 & 95.5 & 17.7 \\
\hline \multicolumn{8}{|l|}{ Women (yr) } \\
\hline $20-29(n=400)$ & 68.0 & 110.8 & 67.1 & 68.7 & 71.0 & 86.8 & 0.8 \\
\hline $30-39(n=195)$ & 71.7 & 113.4 & 72.3 & 80.8 & 65.2 & 88.3 & 1.5 \\
\hline $40-49(n=74)$ & 75.2 & 117.0 & 77.2 & 91.2 & 62.3 & 93.0 & 12.2 \\
\hline $50-59(n=37)$ & 78.6 & 124.5 & 81.4 & 111.7 & 60.6 & 99.9 & 18.9 \\
\hline Total $(n=706)$ & 70.4 & 112.9 & 70.3 & 76.7 & 67.9 & 88.5 & 3.1 \\
\hline
\end{tabular}

Values are presented as mean or percentage.

WC, waist circumference; SBP, systolic blood pressure; DBP, diastolic blood pressure; TG, triglycerides; HDL, high-density lipoprotein; FPG, fasting plasma glucose; MetS, metabolic syndrome.
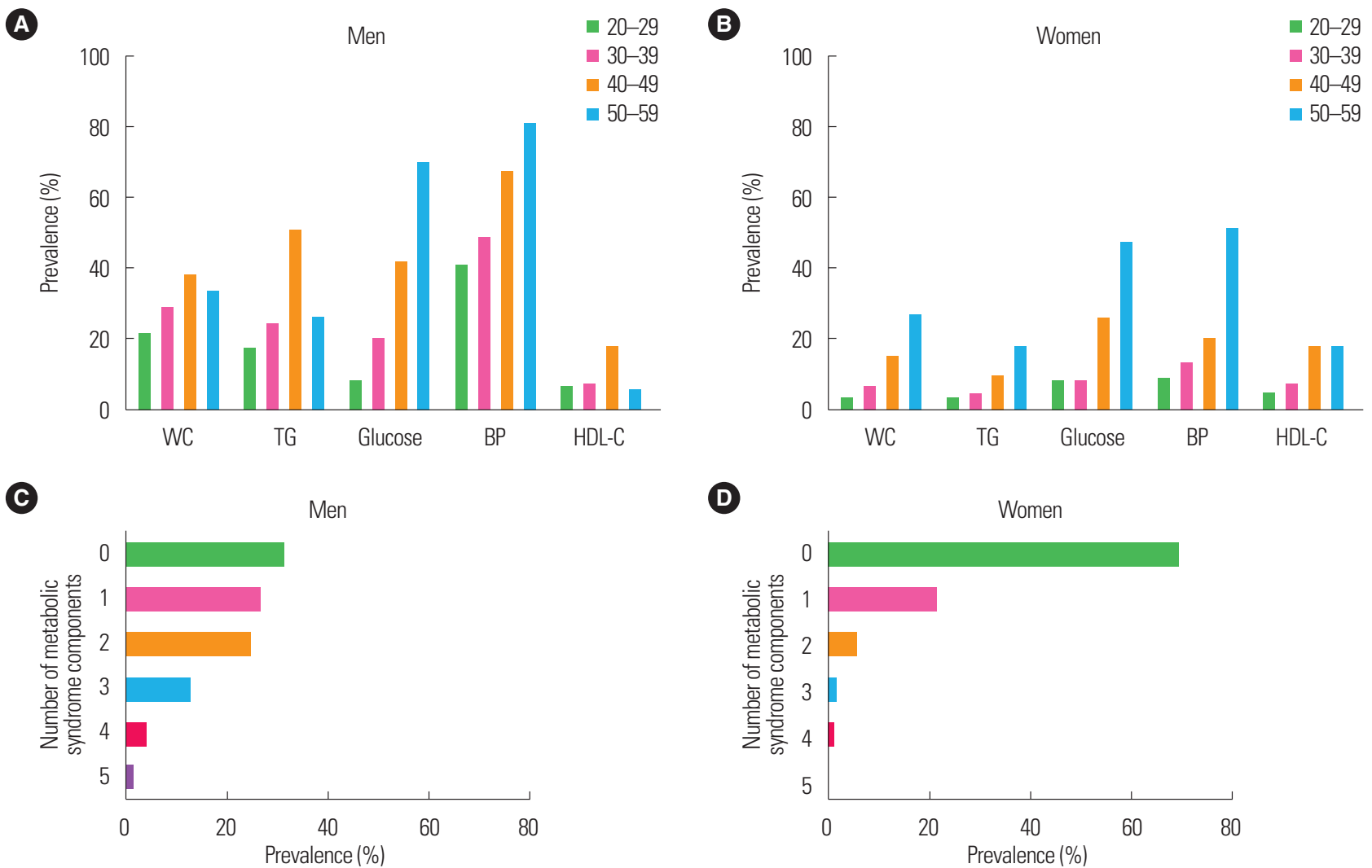

Figure 1. Age group- and sex-specific prevalences of the five individual components of metabolic syndrome in men (A) and women (B). Proportion of subjects with different numbers of metabolic syndrome components in men (C) and women (D). WC, waist circumference; TG, triglycerides; BP, blood pressure; HDL-C, high-density lipoprotein cholesterol.

\section{Nutrient intake}

Table 4 displays the daily nutrient intakes in men and women.

Daily energy intake was 2,004.4 $\pm 499.5 \mathrm{kcal}$ in men and 1,525.0 \pm
$394.5 \mathrm{kcal}$ in women. Men consumed $55.1 \%$ of their energy from carbohydrates, $17.1 \%$ from protein, and $27.7 \%$ from fat. Women consumed $54.8 \%$ of their energy from carbohydrates, $16.6 \%$ from 
Table 4. Body composition, handgrip strength, and nutrient intakes of study participants according to sex

\begin{tabular}{|c|c|c|c|}
\hline Variable & Men & Women & $P$ \\
\hline \multicolumn{4}{|l|}{ Body composition measurement by BIA } \\
\hline Lean body mass $(\mathrm{kg})$ & $53.5 \pm 6.8$ & $36.5 \pm 3.9$ & $<0.001$ \\
\hline Appendicular muscle mass $(\mathrm{kg})$ & $32.0 \pm 4.5$ & $20.9 \pm 2.5$ & $<0.001$ \\
\hline Whole body fat mass (kg) & $17.6 \pm 6.3$ & $16.9 \pm 5.6$ & 0.028 \\
\hline Whole body fat percent (\%) & $23.2 \pm 5.8$ & $29.5 \pm 5.5$ & $<0.001$ \\
\hline Abdominal visceral fat area $\left(\mathrm{cm}^{2}\right)$ & $99.9 \pm 42.0$ & $58.9 \pm 40.2$ & $<0.001$ \\
\hline Abdominal visceral fat area $\geq 100 \mathrm{~cm}^{2}(\%)$ & 47.0 & 16.0 & $<0.001$ \\
\hline Fat $\geq 25 \%$ (men), $\geq 30 \%$ (women) ( $\%$ ) & 35.9 & 45.8 & 0.005 \\
\hline \multicolumn{4}{|l|}{ Handgrip strength } \\
\hline Dominant hand (kg) & $41.2 \pm 7.9$ & $24.1 \pm 4.9$ & $<0.001$ \\
\hline Non-dominant hand (kg) & $38.3 \pm 7.6$ & $22.6 \pm 4.6$ & $<0.001$ \\
\hline$<40 \mathrm{~kg}$ (men), <25 kg (women) (\%) & 42.1 & 56.9 & $<0.001$ \\
\hline \multicolumn{4}{|l|}{ Nutritional intake* } \\
\hline Energy (kcal/day) & $2,004.4 \pm 499.5$ & $1,525.0 \pm 394.5$ & $<0.001$ \\
\hline Carbohydrate (g/day) & $270.5 \pm 73.4$ & $208.2 \pm 62.3$ & $<0.001$ \\
\hline Protein (g/day) & $83.9 \pm 25.0$ & $63.3 \pm 20.6$ & $<0.001$ \\
\hline Fat (g/day) & $60.4 \pm 20.5$ & $48.2 \pm 16.3$ & $<0.001$ \\
\hline Fiber (g/day) & $5.5 \pm 2.6$ & $4.5 \pm 2.4$ & 0.001 \\
\hline \multicolumn{4}{|l|}{ Proportion of total energy intake (\%) } \\
\hline Carbohydrate intake & $55.1 \pm 7.9$ & $54.8 \pm 8.4$ & 0.746 \\
\hline Protein intake & $17.1 \pm 3.3$ & $16.6 \pm 2.9$ & 0.115 \\
\hline Fat intake & $27.7 \pm 6.3$ & $28.6 \pm 7.1$ & 0.282 \\
\hline \multicolumn{4}{|l|}{ Other nutrients } \\
\hline Calcium (mg/day) & $397.3 \pm 233.4$ & $375.3 \pm 209.5$ & 0.385 \\
\hline Sodium (mg/day) & $4,454.1 \pm 2,000.6$ & $3,411.2 \pm 1,838.0$ & $<0.001$ \\
\hline Potassium (mg/day) & $2,697.4 \pm 936.5$ & $2,204.6 \pm 1,074.1$ & $<0.001$ \\
\hline
\end{tabular}

Values are presented as mean \pm standard deviation unless otherwise indicated.

*Nutrient intakes were assessed in 401 participants.

$\mathrm{BIA}$, bioelectrical impedance analysis.

protein, and $28.6 \%$ from fat. Sodium intake was higher in men than in women $(4,454.1 \pm 2,000.6 \mathrm{mg}$ vs. $3,411.2 \pm 1,838.0 \mathrm{mg}, P<$ $0.001)$

\section{DISCUSSION}

In this hospital-based biobank cohort with 1,017 Korean participants aged 20-59 years, the prevalence of metabolic syndrome was $7.6 \%$ overall, with a large difference between the sexes: $17.7 \%$ in men and $3.1 \%$ in women. All individual components of metabolic syndrome were also more prevalent in men than in women. In men, high blood pressure was the most prevalent abnormality (51.4\%), and abdominal obesity and high triglyceride levels were next: $28.3 \%$ and $26.9 \%$, respectively. In women, high blood pres- sure was also the most prevalent abnormality (13.8\%), while hyperglycemia and low HDL cholesterol levels were next: $12.3 \%$ and $7.6 \%$, respectively.

This study revealed that high blood pressure was the most prevalent component of metabolic syndrome in both sexes. However, the second most prevalent abnormality was high triglyceride level in men but low HDL-cholesterol level in women. Similarly, the China Health and Nutrition Survey 2009 indicated that the prevalence of metabolic syndrome according to the revised NCEP-ATP III criteria was $12.4 \%$ in young subjects (18-39 years old), with a large sex difference ( $15.7 \%$ in men and $9.5 \%$ in women) ${ }^{26}$ In that study, the most frequent abnormal component in men was high blood pressure (45.7\%), as in the current study, but the most frequent abnormal component in women was high waist circumference (cutoff $\geq 80 \mathrm{~cm} ; 56.0 \%$ ). After high waist circumference, high blood pressure was the next most prevalent abnormality in women, followed by low HDL-cholesterol (31.0\%).

Interestingly, the prevalence of metabolic syndrome was very low in our cohort. Tran et al. ${ }^{27}$ determined the prevalence of metabolic syndrome using KNHANES data from 2008 to 2013. Using the same diagnostic criteria for metabolic syndrome as those used in our study, they found that the prevalence of metabolic syndrome was $30.8 \%$ in men and $28.9 \%$ in women. The age of the participants and the rates of smoking and alcohol intake were higher in their study than in ours. Furthermore, obesity $\left(\mathrm{BMI} \geq 25 \mathrm{~kg} / \mathrm{m}^{2}\right)$ was more prevalent in the KNHANES than in our biobank cohort (33.2\% vs. $19.0 \%)$.

In our study, current and ex-smokers were extremely rare among Korean women $(0.1 \%$ and $0.8 \%$, respectively). This may have contributed to the significant difference in incidence of high blood pressure between the sexes: $51.4 \%$ in men but only $13.8 \%$ in women. Lower sodium intake in women also may have contributed to their low incidence of hypertension in the present study. ${ }^{28}$ Furthermore, previous KNHANES data indicated that metabolic syndrome was more prevalent in housewives than in female office workers. ${ }^{9}$ These socioenvironmental factors likely contributed to the low prevalence of metabolic syndrome among women in this study. Other Asian surveys and meta-analyses have also shown that prevalence of metabolic syndrome tends to be higher in men than in women in young populations. ${ }^{29,30}$ 
According to national statistical data on Korean adults, total daily caloric intake increased from 2,196 kcal in 1998 to $2,457 \mathrm{kcal}$ in 2010-2012 in men. In stark contrast, it decreased from 1,748 kcal to $1,721 \mathrm{kcal}$ over the same period in women. ${ }^{31}$ In our study, total caloric intake was slightly lower than that in the Korean national data. In addition, macronutrient composition was very similar to that recommended by the NCEP-ATP III for therapeutic dietary changes. ${ }^{32}$ An intervention study revealed that it was beneficial for subjects with metabolic syndrome to reduce their total daily caloric intake and to follow the NCEP-ATP III diet. ${ }^{33}$

In our study, handgrip strength in the dominant hand was $41.2 \pm$ $7.9 \mathrm{~kg}$ in men and $24.1 \pm 4.9 \mathrm{~kg}$ in women. These values were equivalent to those from a previous study in 335 healthy young European adults, showing $44.8 \pm 6.6 \mathrm{~kg}$ in men and $27.7 \pm 4.3 \mathrm{~kg}$ in women. ${ }^{34}$ Muscle strength assessed by handgrip strength is linked with various health parameters, including physical performance, cardiorespiratory fitness, and mortality. ${ }^{35,36}$ In a study of $>1,000$ Koreans, relative skeletal muscle mass was negatively associated with development of metabolic syndrome. ${ }^{37} \mathrm{~A}$ recent report on the US NHANES 2011-2012 indicated that handgrip strength was also linked with cardiovascular risk factors. ${ }^{38}$ Considering that both body composition and grip strength are associated with cardiometabolic risk ${ }^{39}$, the combined measurement of body composition and muscle strength could provide comprehensive information on cardiometabolic risk.

Previous studies have had inconsistent results regarding the association between $\mathrm{BMI}$ and handgrip strength. ${ }^{40}$ This discrepancy may arise from the fact that BMI cannot distinguish between muscle and fat mass. In this regard, our cohort study had the advantage of including a detailed body composition assessment, which revealed that handgrip strength was associated positively with muscle mass and negatively with body fat percentage.

Our study had several limitations and strengths. First, this hospital-based cohort may not represent the general population. However, compared with the KNHANES, we enrolled a relatively young group of participants, which allowed us to identify a large difference between male and female subjects. This unique finding has provided novel insights into the pathogenesis of metabolic syndrome. Second, the KNHANES studies are cross-sectional rather than longitudinal prospective studies, and blood samples are not collected. In contrast, we obtained serum and plasma blood samples, purified RNA, and buffy coats for DNA information collection and future analysis. Genetic analyses will provide information on candidate genes that are associated with increased risk of cardiometabolic disorders. Third, we conducted OGTTs to investigate glucose homeostasis comprehensively. Blood samples after glucose loading are highly valuable and are not available in the KNHANES. In future work, we will measure biomarkers induced by glucose loading, such as gut hormones.

In conclusion, this metabolic syndrome-Biobank-SNUBH survey was a well-organized prospective cohort study focused on metabolic phenotypes and body composition analysis. The "Westernization" of lifestyle, including high-fat and high-calorie diet intake and physical inactivity, has had significant adverse health consequences in South Korea. Metabolic syndrome is increasing in both young people and adults in Korea ${ }^{8,11}$, and is becoming a socioeconomic burden. Given this situation, it was timely to establish a solid cohort from the young-to-middle-aged population to study metabolic syndrome. Although our cohort was not community-based, hospital-based cohorts have several advantages, including availability of detailed information about comorbidities, convenience of follow-up, and ease of continuing data collection. Furthermore, we included socially active young-to-middle-aged adults, and this homogeneous study population will facilitate our understanding of cardiometabolic diseases. Future longitudinal follow-up and collaboration with other hospital-based cohort studies will enable us to investigate the risk factors that predict cardiometabolic events in young and middle-aged Koreans.

\section{CONFLICTS OF INTEREST}

The authors declare no conflict of interest.

\section{ACKNOWLEDGMENTS}

This study was supported by grants from the Seoul National University Bundang Hospital research fund (15-2015-003, 10-2016002).

The authors acknowledge the research nurses and all study participants. 


\section{SUPPLEMENTARY MATERIALS}

Supplementary Table 1. Baseline examination in this hospitalbased biobank cohort.

Supplementary Table 2. Components of metabolic syndrome according to parity in women.

They can be found via https://doi.org/10.7570/jomes.2019.28. 2.118 .

\section{REFERENCES}

1. Isomaa B, Almgren P, Tuomi T, Forsén B, Lahti K, Nissén M, et al. Cardiovascular morbidity and mortality associated with the metabolic syndrome. Diabetes Care 2001;24:683-9.

2. Gami AS, Witt BJ, Howard DE, Erwin PJ, Gami LA, Somers VK, et al. Metabolic syndrome and risk of incident cardiovascular events and death: a systematic review and meta-analysis of longitudinal studies. J Am Coll Cardiol 2007;49:403-14.

3. International Diabetes Federation. The IDF consensus worldwide definition of the metabolic syndrome. Brussels: International Diabetes Federation; 2006.

4. Alberti KG, Zimmet PZ. Definition, diagnosis and classification of diabetes mellitus and its complications. Part 1: diagnosis and classification of diabetes mellitus provisional report of a WHO consultation. Diabet Med 1998;15:539-53.

5. Grundy SM, Cleeman JI, Daniels SR, Donato KA, Eckel RH, Franklin BA, et al. Diagnosis and management of the metabolic syndrome: an American Heart Association/National Heart, Lung, and Blood Institute Scientific Statement. Circulation 2005;112:2735-52.

6. Alberti KG, Zimmet P, Shaw J. Metabolic syndrome: a new world-wide definition. A Consensus Statement from the International Diabetes Federation. Diabet Med 2006;23:469-80.

7. Hong AR, Lim S. Clinical characteristics of metabolic syndrome in Korea, and its comparison with other Asian countries. J Diabetes Investig 2015;6:508-15.

8. Lim S, Shin H, Song JH, Kwak SH, Kang SM, Won Yoon J, et al. Increasing prevalence of metabolic syndrome in Korea: the Korean National Health and Nutrition Examination Survey for 1998-2007. Diabetes Care 2011;34:1323-8.
9. Kim E, Oh SW. Gender differences in the association of occupation with metabolic syndrome in Korean adults. Korean J Obes 2012;21:108-14.

10. Seo MH, Kim YH, Han K, Jung JH, Park YG, Lee SS, et al. Prevalence of obesity and incidence of obesity-related comorbidities in Koreans based on National Health Insurance Service Health Checkup Data 2006-2015. J Obes Metab Syndr 2018;27:46-52.

11.Lim S, Jang HC, Park KS, Cho SI, Lee MG, Joung H, et al. Changes in metabolic syndrome in American and Korean youth, 1997-2008. Pediatrics 2013;131:e214-22.

12.Aguilar M, Bhuket T, Torres S, Liu B, Wong RJ. Prevalence of the metabolic syndrome in the United States, 2003-2012. JAMA 2015;313:1973-4.

13. Lutsey PL, Steffen LM, Stevens J. Dietary intake and the development of the metabolic syndrome: the Atherosclerosis Risk in Communities study. Circulation 2008;117:754-61.

14. Ford ES, Kohl HW 3rd, Mokdad AH, Ajani UA. Sedentary behavior, physical activity, and the metabolic syndrome among U.S. adults. Obes Res 2005;13:608-14.

15. Choi JH, Woo HD, Lee JH, Kim J. Dietary patterns and risk for metabolic syndrome in Korean women: a cross-sectional study. Medicine (Baltimore) 2015;94:e1424.

16. Nam JY, Kim J, Cho KH, Choi Y, Choi J, Shin J, et al. Associations of sitting time and occupation with metabolic syndrome in South Korean adults: a cross-sectional study. BMC Public Health 2016;16:943.

17. Kim J, Choi YH. Physical activity, dietary vitamin C, and metabolic syndrome in the Korean adults: the Korea National Health and Nutrition Examination Survey 2008 to 2012. Public Health 2016;135:30-7.

18. Yang EJ, Lim S, Lim JY, Kim KW, Jang HC, Paik NJ. Association between muscle strength and metabolic syndrome in older Korean men and women: the Korean Longitudinal Study on Health and Aging. Metabolism 2012;61:317-24.

19. Lim S, Kim JH, Yoon JW, Kang SM, Choi SH, Park YJ, et al. Sarcopenic obesity: prevalence and association with metabolic syndrome in the Korean Longitudinal Study on Health and Aging (KLoSHA). Diabetes Care 2010;33:1652-4.

20.Hori A, Nanri A, Sakamoto N, Kuwahara K, Nagahama S, 
Kato N, et al. Comparison of body mass index, waist circumference, and waist-to-height ratio for predicting the clustering of cardiometabolic risk factors by age in Japanese workers: Japan Epidemiology Collaboration on Occupational Health study. Circ J 2014;78:1160-8.

21. Hu FB, Manson JE, Stampfer MJ, Colditz G, Liu S, Solomon $\mathrm{CG}$, et al. Diet, lifestyle, and the risk of type 2 diabetes mellitus in women. N Engl J Med 2001;345:790-7.

22. Shin S, Park E, Sun DH, You TK, Lee MJ, Hwang S, et al. Development and evaluation of a web-based computer-assisted personal interview system (CAPIS) for open-ended dietary assessments among Koreans. Clin Nutr Res 2014;3:115-25.

23. Lee DH, Park KS, Ahn S, Ku EJ, Jung KY, Kim YJ, et al. Comparison of abdominal visceral adipose tissue area measured by computed tomography with that estimated by bioelectrical impedance analysis method in Korean subjects. Nutrients 2015;7:10513-24.

24. Shiiya T, Nakazato M, Mizuta M, Date Y, Mondal MS, Tanaka $\mathrm{M}$, et al. Plasma ghrelin levels in lean and obese humans and the effect of glucose on ghrelin secretion. J Clin Endocrinol Metab 2002;87:240-4.

25. Schlüssel MM, dos Anjos LA, de Vasconcellos MT, Kac G. Reference values of handgrip dynamometry of healthy adults: a population-based study. Clin Nutr 2008;27:601-7.

26. Xi B, He D, Hu Y, Zhou D. Prevalence of metabolic syndrome and its influencing factors among the Chinese adults: the China Health and Nutrition Survey in 2009. Prev Med 2013;57:86771.

27. Tran BT, Jeong BY, Oh JK. The prevalence trend of metabolic syndrome and its components and risk factors in Korean adults: results from the Korean National Health and Nutrition Examination Survey 2008-2013. BMC Public Health 2017;17:71.

28. Cook NR, Cutler JA, Obarzanek E, Buring JE, Rexrode KM, Kumanyika SK, et al. Long term effects of dietary sodium reduction on cardiovascular disease outcomes: observational follow-up of the trials of hypertension prevention (TOHP). BMJ 2007;334:885-8.

29. Binh TQ Phuong PT, Nhung BT, Tung do D. Metabolic syndrome among a middle-aged population in the Red River Delta region of Vietnam. BMC Endocr Disord 2014;14:77.

30.Li R, Li W, Lun Z, Zhang H, Sun Z, Kanu JS, et al. Prevalence of metabolic syndrome in Mainland China: a meta-analysis of published studies. BMC Public Health 2016;16:296.

31. Ha YS. Trends in energy intake and food sources of energy intake among Korean adults. Public Health Wkly Rep 2014;7: 829-37.

32. Expert Panel on Detection, Evaluation, and Treatment of High Blood Cholesterol in Adults. Executive summary of the third report of the National Cholesterol Education Program (NCEP) expert panel on detection, evaluation, and treatment of high blood cholesterol in adults (Adult Treatment Panel III). JAMA 2001;285:2486-97.

33. Muzio F, Mondazzi L, Sommariva D, Branchi A. Long-term effects of low-calorie diet on the metabolic syndrome in obese nondiabetic patients. Diabetes Care 2005;28:1485-6.

34. Montalcini T, Migliaccio V, Yvelise F, Rotundo S, Mazza E, Liberato A, et al. Reference values for handgrip strength in young people of both sexes. Endocrine 2013;43:342-5.

35. Ling CH, Taekema D, de Craen AJ, Gussekloo J, Westendorp RG, Maier AB. Handgrip strength and mortality in the oldest old population: the Leiden 85-plus study. CMAJ 2010;182: 429-35.

36.Zaqout M, Michels N, Bammann K, Ahrens W, Sprengeler O, Molnar D, et al. Influence of physical fitness on cardio-metabolic risk factors in European children: the IDEFICS study. Int J Obes (Lond) 2016;40:1119-25.

37. Park BS, Yoon JS. Relative skeletal muscle mass is associated with development of metabolic syndrome. Diabetes Metab J 2013;37:458-64.

38. Lawman HG, Troiano RP, Perna FM, Wang CY, Fryar CD, Ogden CL. Associations of relative handgrip strength and cardiovascular disease biomarkers in U.S. adults, 2011-2012. Am J Prev Med 2016;50:677-83.

39. Lim S, Meigs JB. Ectopic fat and cardiometabolic and vascular risk. Int J Cardiol 2013;169:166-76.

40. Gale CR, Martyn CN, Cooper C, Sayer AA. Grip strength, body composition, and mortality. Int J Epidemiol 2007;36: 228-35. 